\title{
Similitudes y diferencias en el déficit lingüistico de dos casos de huntington con variables médicas similares
}

\author{
VÍCTOR MANUEL MARTín-SÁNCHEZ \\ Universidad de Cádiz \\ Facultad de Filosofia y Letras \\ Avda. Dr. Gómez Ulla, s/n \\ 11003 Cádiz \\ E-mail: victor.martin@uca.es
}

\section{SIMILITUDES Y DIFERENCIAS EN EL DÉFICIT LINGÜÍSTICO DE DOS CASOS DE HUNTING- TON CON VARIABLES MÉDICAS SIMILARES}

RESUMEN: Muchos manuales médicos proporcionan información para diagnosticar pacientes y delimitar su estadio de evolución; sin embargo, la información de estos informes no siempre es útil para los logopedas por ofrecer escasos datos lingüisticos del paciente. A esto se suma la problemática de que diferentes pacientes con el mismo diagnóstico no tienen por qué presentar los mismos sintomas. Para solucionar este problema proponemos que exista la figura de un experto en el lenguaje que elabore un informe lingüístico clínico de cada paciente. Los objetivos son conocer el comportamiento lingüístico de dos pacientes con huntington que se encuentren en el mismo estadio de evolución; extraer datos lingüisticos que no se presentan en los informes neurológicos y establecer una comparación para clarificar las semejanzas y diferencias entre ambos. Por ello, proporcionamos un análisis lingüístico clínico, a través de la conversación espontánea y guiada, de dos pacientes con huntington con las mismas variables médicas y sociolingüisticas.

PALABRAS ClAVES: Lingüistica clínica; Enfermedad de Huntington; Estudio de caso; Evaluación; Sintomatologia lingüistica.

SUMARIO: 1. Introducción. 2. Hipótesis y objetivos. 3. Huntington. 4 Metodología. 5. Análisis de los casos. 6. Conclusiones

\section{SIMILARITIES \\ DIFFERENCES IN THE \\ LINGUISTIC DEFICIT OF TWO HUNTINGTONIAN CASES \\ WITH SIMILAR MEDICAL VARIABLES}

ABSTRACT: Many medical manuals provide information to diagnose patients and define their stage of evolution. However, the information in these reports is not always useful for speech therapists because they offer scarce linguistic data about the patient. This is compounded by the problem that different patients with the same diagnosis do not have to present the same symptoms. To solve this problem we propose the existence of a figure of an expert in the language that develops a clinical linguistic report of each patient. The objectives are to know the linguistic behavior of two huntington patients who are in the same stage of evolution; extract linguistic data that are not presented in the neurological reports and establish a comparison to clarify the similarities and differences between both. Therefore, we provide a clinical linguistic analysis, through spontaneous and guided conversation, of two huntingtonian patients with the same medical and sociolinguistic variables.

KEY WORDS: Clinical linguistics; Huntington's disease; Case study; Assessment; Linguistic symptomatology.

SUMMARY: 1 . Introduction. 2. Hypotheses and objectives. 3. Huntington. 4. Methodology. 5. Analysis of the cases. 6 . Conclusions.

\section{SIMILITUDES ET DIFFÉRENCES DANS LE DÉFICIT LINGUISTIQUE DE DEUX CAS DE HUNTINGTON AYANT DES VARIABLES SIMILAIRES}

RÉSUMÉ: De nombreux manuels médicaux fournissent des informations pour diagnostiquer les patients et définir leur stade d'évolution. Toutefois, les informations de ces rapports ne sont pas toujours utiles pour les orthophonistes, car ils offrent peu de données linguistiques sur le patient. À cela s'ajoute le problème suivant : différents patients avec le même diagnostic ne présentent pas nécessairement les mêmes symptômes. Pour résoudre ce problème, nous proposons l'introduction de la figure d'un expert en langage afin de développer un rapport linguistique clinique de chaque patient. Les objectifs sont de connaitre le comportement linguistique de deux patients de Huntington qui sont au même stade d'évolution, d'extraire des données linguistiques qui ne sont pas présentes dans les rapports neurologiques et d'établir une comparaison afin de clarifier les similitudes et les différences entre les deux. C'est pourquoi nous fournissons une analyse linguistique clinique, à travers la conversation spontanée et guidée, de deux patients de Huntington présentant les mêmes variables médicales et sociolinguistiques.

MOTS CLÉS: Linguistique clinique La maladie de Huntington; Étude de cas ; Évaluation ; Symptomatologie linguistique.

\section{SOMMAIRE:}

1. Introduction . 2. Hypothèses et objectifs . 3. Huntington . 4. Méthodologie . 5. Analyse des cas . 6. Conclusions .
Fecha de Recepción Fecha de Revisión Fecha de Aceptación Fecha de Publicación 


\title{
Similitudes y diferencias en el déficit lingüístico de dos casos de huntington con variables médicas similares
}

\author{
VÍCTOR MANUEL MARTíN-SÁNCHEZ
}

\section{INTRODUCCIÓN}

En la actualidad, se considera generalmente que las diferentes enfermedades y sus diagnósticos están lo suficientemente acotados para no generar dudas entre los profesionales que las tratan. Además, esta creencia se ve respaldada por la ingente cantidad de manuales médicos ${ }^{1}$ donde podemos encontrar unas directrices muy claras de las diferentes patologias y de qué datos deben ser tenidos en cuenta para discernir entre los diversos diagnósticos. No obstante, en la práctica encontramos problemas, puesto que no todos los pacientes se ajustan a lo esperado.

El principal motivo es la variabilidad del déficit (ya sea lingüístico, motor, comportamental o cognitivo) entre distintos pacientes como entre diferentes momentos del mismo sujeto. Con esta afirmación hacemos referencia a que es muy común encontrar pacientes que compartan un mismo diagnóstico pero que la sintomatología sea muy dispar. Lo mismo ocurre, lógicamente, con una misma enfermedad durante el tránsito del paciente por los estadios de evolución, como el caso del huntington. Llegados a este punto, cabe preguntarse si realmente es posible delimitar fronteras exactas entre patologías de un mismo grupo - como los trastornos motores - o saber el momento en el que un paciente con una enfermedad neurodegenerativa transita las lindes de una fase a la siguiente basándonos en los sintomas.

A estos problemas se enfrentan los profesionales de áreas cientificas encargados de describir o identificar el comportamiento general de una patología; sin embargo, cada uno desde un punto de vista y con unos objetivos distintos. Por ejemplo, los neurólogos, neuropsicólogos y psiquiatras se encargan de establecer un análisis de la sintomatología motora, cognitiva y comportamental de cada paciente a la vez del diagnóstico definitivo de su patología, mientras que los lingüistas desempeñan la labor de describir el comportamiento lingüístico de los enfermos. Por último, encontramos a los rehabilitadores - como son los fisioterapeutas y los logopedas-cuya labor es utilizar los datos proporcionados por los informes diagnósticos para comenzar un programa de rehabilitación adecuado a cada caso. No obstante, en la gran mayoria de los casos, los hospitales no cuentan con la labor de lingüistas. Por lo tanto, los logopedas deben trabajar tan solo con el diagnóstico proporcionado por el neurólogo, que es insuficiente puesto que carece de datos sobre las habilidades comunicativas del paciente, además de que estas no siempre son fáciles de prever por el diagnóstico asignado.

\footnotetext{
${ }^{1}$ Como ejemplo se puede mencionar uno de los más famosos, como es el Harrison. Manual de medicina (2017).
} 
En la elaboración de cualquier estudio resulta necesario llevar a cabo una búsqueda bibliográfica sobre los estudios científicos realizados sobre la temática que se desea tratar. Esto nos ayuda a situarnos mejor en el momento de comenzar con el estudio y a no cometer los mismos errores o llegar a conclusiones de anteriores investigadores. No obstante, no siempre podemos encontrar precedentes, debido a múltiples razones. En nuestro caso, encontramos una serie de dificultades para esta labor:

1) Las disciplinas de análisis desde las que partimos son la lingüística clínica y la logopedia. Ambas son originarias del pasado siglo y todavía no han tenido un desarrollo tan avanzado como otras que llevan siendo estudiadas durante mucho más tiempo.

2) La enfermedad estudiada es el huntington, que es calificada como enfermedad rara, por lo que es menos considerada que otras con mayor prevalencia como el párkinson, el alzhéimer, la dislexia o el trastorno por déficit de atención e hiperactividad.

3) Los profesionales que se han preocupado por describir y estudiar la patología proceden de ámbitos de conocimiento diferentes al lingüístico, como son la neurología, la psicología o la psiquiatría.

Por todo ello, los artículos científicos que presentamos son escasos y están relacionados con el trabajo que realizamos aquí sin embargo, ninguno de ellos se centra específicamente en aspectos lingüísticos o comunicativos en pacientes sintomáticos de huntington.

En primer lugar, y siendo el artículo más relacionado con el tema de este proyecto, aludimos el estudio "A systematic linguistic profile of spontaneous narrative speech in pre-sintomatic and early stage Huntington's disease" (Hinzen et al., 2018). Como mencionamos en la introducción de este trabajo, se trata de un estudio que sigue una metodología parecida a la que utilizamos aquí; no obstante, se evalúan muchos menos aspectos. Además, la cantidad de pacientes analizados es mayor, ya que lo que se intenta es construir un perfil lingüístico de enfermos de huntington presíntomáticos en un nivel más general. Por el contrario, nuestro estudio se cierne a una comparación del análisis comunicativo de dos pacientes sintomáticos. Lo que nos parece más interesante es que sea uno de los pocos estudios a nivel mundial que trata de establecer un perfil lingüístico. De este modo, se toman los datos neurológicos y neuropsicológicos como base en el estudio lingüístico, siendo este último su centro de interés.

En segundo lugar, nos parece relevante destacar el "Estudio neuropsicológico de la enfermedad de Huntington en familias de Antioquia, Colombia" (Arango-Lasprilla et al., 2003), debido a su gran similitud metodológica. Como vemos en su título, se trata de un estudio neuropsicológico general, no puramente lingüístico; no obstante, se han evaluado aspectos lingüisticos y se han tenido en cuenta en los resultados obtenidos, puesto que, en 
la descripción sintomatológica de los pacientes, podemos encontrar dificultades en la fluidez verbal y en la retención de información y poca agilidad articulatoria. Además, se trata de un estudio de treinta y tres sujetos pertenecientes a siete familias, mientras que nosotros hemos realizado un estudio de caso de dos pacientes.

Lo más acentuable es la evaluación de ciertos aspectos lingüísticos a través de baterías como el CERAD y pruebas neuropsicológicas adicionales. Algunos de ellos serán evaluados también en nuestro trabajo. Asimismo, dentro del CERAD, encontramos cinco tareas lingüísticas:

1) la fluidez verbal, en la que el paciente debe nombrar todos los animales que conozca en un minuto;

2) el test de denominación, donde el entrevistador pregunta por el nombre de veinte dibujos, cuya frecuencia va disminuyendo;

3) la memorización de una lista de palabras, en la que el paciente debe leer diez palabras en dos o tres segundos y tratar de recordar el máximo número posible de forma inmediata;

4) el recuerdo de la lista de palabras anterior tras haber realizado una actividad intermedia, $\mathrm{y}$

5) el reconocimiento de la lista de palabras, donde el paciente se encuentra veinte palabras, diez de las cuales conoce de la tarea de memorización y otras diez que no aparecieron en ella, y debe tratar de localizar las conocidas.

Por otro lado, dentro de las pruebas neuropsicológicas adicionales también vemos interesantes las siguientes tareas:

1) el test de frases, que consiste en que el entrevistador pronuncie tres frases cortas para que el paciente las repita, y

2) el test de fluidez verbal fonológica, en el que el paciente debe decir el máximo número de palabras que empiezan por una letra, en este caso la efe.

Tras la lectura de este artículo, pudimos observar que, pese a que la mitad de los test eran de carácter lingüístico, los resultados se centraron en aspectos cognitivos, como la memoria o las funciones ejecutivas. Además, el estudio no se basaba en la descripción sintomatológica de un grupo de personas afectadas, sino en el contraste entre los resultados de los afectados frente a un grupo de familiares sanos, lo cual difiere de nuestras intenciones.

En tercer lugar, vamos a hablar del trabajo "Huntington's disease in two New Britain families" (Scrimgeour, 1980). También se trata de un estudio de casos, pero, al igual que el anterior, sobre miembros de familias afectados por la enfermedad de Huntington. Concretamente, el autor estudia dos familias de Melanesia y establece una comparación entre los sintomas que 
ambas presentan. No obstante, en la discusión final, afirma que las dos familias podrian estar emparentadas.

Lo que nos parece relevante de este estudio es que se nos proporcionan más datos contextuales de cada individuo: datos demográficos, algún dato clínico y sintomas de cada sujeto, de entre los cuales podemos destacar alguno lingüístico, como la disartria, la escasa conversación o las perseveraciones. Esto entronca levemente con el trabajo que presentamos aquí, ya que la descripción lingüística es pobre.

En cuarto lugar, encontramos un nuevo artículo de otro estudio sobre una familia llamado "Estudio de una familia con corea de Huntington en Cañete" (Cuba y Torres, 1990). En este trabajo, vemos un estudio de casos sobre un grupo de personas pertenecientes a la misma familia procedente de El Valle del Cañete. Sin embargo, no se han tenido en cuenta la sintomatología lingüística, ya que el autor solo hace referencia a los movimientos involuntarios de tipo coreico, las manifestaciones psicopatológicas y la demencia.

Quizá el mayor punto en común con lo que nos compete es que el trabajo se realizó a través de entrevistas personalizadas en el Valle del Cañete. Algo similar a lo que hemos realizado en la provincia de Cádiz con los pacientes en cuestión.

Por último, presentamos el quinto estudio de relativa semejanza a este proyecto llamado "Enfermedad de Huntington. Reporte de dos casos de una familia hondureña y revisión de la literatura" (Hesse et al, 2006). Aquí encontramos una investigación sobre dos casos concretos de personas que padecen el huntington. Nos llama la atención que vemos una presencia escasa de aspectos lingüísticos y se inscriben dentro de la sintomatología cognitiva (en el caso de la comprensión) y motora (en el caso de la producción deficiente de sonidos).

En cuanto a las semejanzas con nuestro estudio, podemos mencionar que se presentan datos clínicos de los casos (aunque algunos son muy personales) y una gran descripción de la enfermedad como revisión de la literatura cientifica, que aclara y contextualiza la investigación.

\section{HIPÓTESIS Y OBJETIVOS}

Teniendo en cuenta todo lo anterior y con la intención de resolver esta carencia en el actual ámbito médico, proponemos como hipótesis para el presente estudio la necesidad de completar el diagnóstico neurológico con una evaluación comunicativa elaborada por un profesional del lenguaje. Concretamente, ese profesional deberá poseer amplios conocimientos tanto literario como culturales y, sobre todo, lingüísticos, de la lengua hablada por los pacientes que se pretendan analizar. Este último aspecto resulta ser el más relevante y podría concretarse más al tener en cuenta la variabilidad del uso de una lengua geográficamente. Por ejemplo, si el estudio se realiza a personas de la provincia de Extremadura, serán de vital importancia los 
conocimientos sobre el dialecto extremeño para discernir entre el lenguaje patológico y el dialectal. Cabe añadir también que, debido a la gran cantidad de migración de hoy en día y al contacto entre personas de culturas, dialectos y lenguas distantes entre sí, se requiere un conocimiento sociolingüistico más amplio para la fiabilidad de los datos obtenidos.

Para llevar a cabo el estudio, nos centraremos en el cumplimiento de los siguientes objetivos:

- conocer el comportamiento lingüístico de dos pacientes con huntington que se encuentren en el mismo estadio de evolución;

- extraer datos lingüísticos que no se presentan en los informes neurológicos, $\mathrm{y}$

- establecer una comparación para clarificar las semejanzas y diferencias entre ambos.

\section{HunTINGTON}

A continuación, desarrollaremos una descripción general de la patología que estudiamos para mayor comprensión del estudio realizado. Lo primero que vamos a plantearnos aquí es la manera de definir la enfermedad de Huntington y el motivo por el que se la llama así, ya que esta información nos proporcionará una idea general de la misma. Por ello, partimos de que esta enfermedad no facilita un análisis etimológico del término, como puede ser el caso de la dislexia, la disgrafia, la hipotonía o la afasia, de las cuales podemos extraer las raíces griegas y, por consecuente, una gran cantidad de información sobre las mismas. Por el contrario, el huntington, al igual que el párkinson, el alzhéimer o los síndromes de Down y Williams, es una patología cuyo nombre viene dado por atribución científica a un autor concreto, que en este caso es George Huntington (1850-1916). Este hombre fue un médico graduado en la Universidad de Columbia y que fue invitado en el año 1872 a la Meigs and Mason Academy de Middleport, en Ohio. Alli compartió su trabajo titulado Sobre la corea, en el que presentó una descripción de la evolución de la enfermedad a través de varias generaciones en la familia Wells (Franch Valverde, 1993; García de Yébenes, 2006). Por ser uno de los pioneros en presentar un estudio tan detallado de esta patología, se la conoce como huntington o enfermedad de Huntington. No obstante, se la ha conocido de muchas formas. Franch Valverde (1993) nos las unifica de la siguiente manera:

- corea mayor,

- corea crónica,

- corea hereditaria,

- corea crónica progresiva,

- corea crónica hereditaria,

- corea crónica degenerativa, 
- corea crónica del adulto,

- corea crónica progresiva hereditaria,

- demencia coreica,

- corea de Huntington,

- enfermedad de Huntington, y

- baile de San Vito hereditario.

Es posible apreciar que ha habido una predisposición a acuñar un término que haga referencia a los síntomas que manifiesta dicha enfermedad. Concretamente, el más recurrente es el término "corea", que proviene del griego clásico xopєia 'baile, danza'. Esto se debe a que, al ser un trastorno motor, el movimiento coreico — similar a un baile — es el rasgo más notorio en los pacientes. Sin embargo, el término más aceptado desde los años 80 es el de enfermedad de Huntington (Roos, 2010) y, recientemente, ha llegado a la lengua común como "huntington".

Su etiología es de corte genético. Para ser más específicos, el huntington es provocado por una expansión anormal del triplete compuesto por citosina, adenina y guanina (CAG) en la secuencia del gen HD. Esto se debe a una mutación del brazo corto del cromosoma 4p16.3 en dicho gen (Benítez Burraco, 2008; Roos, 2010), donde, a su vez, se codifica la proteina huntingtina, que se desarrolla en diferentes regiones cerebrales y otros tejidos corporales (citado por Gusella y McDonald en Benítez, 2008).

Con respecto al número de tripletes de CAG que podemos encontrar, tenemos que decir que depende de cada individuo y el nivel de afectación. En general todo individuo posee esta serie de tripletes; sin embargo, para que la enfermedad se manifieste es necesario superar una cantidad de apariciones del mismo en el ADN. En una persona sana, podemos encontrar entre 11 y 35 repeticiones, y se considera que a partir de las 38 se manifiestan los síntomas (Peña-Casanova y Robles Bayón, 2002). Asimismo, sabemos que cuanto mayor sea el número de apariciones de CAG, antes de manifestarán los síntomas y de forma más agresiva.

Por lo general, el huntington es una patología que se revela entre los 30 y los 50 años de vida del individuo (Peña-Casanova y Robles Bayón, 2002; García de Yébenes, 2006; Sarason y Sarason, 2006), pero también existen las variantes juveniles, cuya aparición puede ser antes de los 20, y la senil, que tras los 55 años de edad, empiezan a apreciarse los sintomas.

Todo experto en huntington, piensa en tres características básicas que generan una idea acerca de la enfermedad. La primera es la neurodegeneración, ya que es una patologia que consiste en la muerte de regiones neuronales gabérgicas del núcleo caudado y se va extendiendo a diferentes zonas cerebrales a lo largo del proceso degenerativo (Benitez Burraco, 2009).

La segunda es su carácter hereditario, puesto que se trata de una enfermedad que se traspasa de padres a hijos por la expresión de su gen autosómico dominante (Roos, 2010). En este caso, conviene saber si la herencia se produce por parte de madre o de padre, debido a que en los casos en los que 
proviene del padre hay una mayor posibilidad de que el número de repeticiones de CAG en el ADN sea mayor y, por lo tanto, existen más posibilidades de que se dé un caso de la variante juvenil (Peña-Casanova y Robles Bayón, 2002).

Y la tercera es su evolución y subdivisión de la misma en diferentes estadios. Algunos autores la subdividen en cinco y otros en tres. Nosotros tomaremos como referencia la segunda de las dos siguiendo a Roos (2010). En el primer estadio los pacientes comienzan a experimentar síntomas de alarma, como la corea o la disartria. En cambio, en el segundo se vuelven más agresivos y este carácter aumenta gradientemente hasta el tercer estadio, donde pierden totalmente su independencia y la capacidad de realizar las tareas diarias. Se data de quince años aproximadamente entre el comienzo de la aparición de los síntomas hasta la muerte del paciente (Franch Valverde, 1993), aunque determinar el tiempo no suele ser fácil, puesto que el desarrollo de la enfermedad en cada paciente es distinto. En nuestra experiencia en consulta, algunos pacientes han superado los quince años y otros no han logrado vivir tanto tiempo debido a la muerte prematura por alguno de los sintomas.

Por último, nos gustaría ofrecer una descripción de los principales síntomas que se han aportado en la bibliografia sobre el huntington en la Tabla 1. Cabe destacar que en la mayoría de las referencias hemos hallado información sobre la tríada sintomatológica, que es la compuesta por problemas motores, cognitivos y de comportamiento. No obstante, vamos a añadir la cuarta rama recopilando información de algún estudio neuropsicológico que ha evaluado tangencialmente aspectos lingüisticos junto con otros estudios elaborados en cooperación entre lingüistas y neuropsicólogos.

\begin{tabular}{|l|l|}
\hline \multicolumn{1}{|c|}{ Sintomatología motora } & \multicolumn{1}{c|}{ Sintomatologia cognitiva } \\
\hline $\begin{array}{l}\text { Corea, distonía, tics, mioclonias, ataxia, dis- } \\
\text { fagia, hipocinesia, bradicinesia, hipercine- } \\
\text { sia, acinesia }{ }^{2} \text {, variante de Westphal, rigidez } \\
\begin{array}{l}\text { yalteraciones de movilidad ocular (Garcia de } \\
\text { Yébenes, 2006; Peña-Casanova y Robles Ba- } \\
\text { yón, 2002; Roos, 2010). }\end{array}\end{array}$ & $\begin{array}{l}\text { Amnesia, apraxia, poca flexibilidad mental, } \\
\text { bradifrenia o bradipsiquia, problemas de } \\
\text { atención y de aprendizaje, entre otros } \\
\text { (Arango-Lasprilla, Iglesias-Dorado y Lopera, } \\
\text { 2003; Garcia de Yébenes, 2006; Peña-Casa- } \\
\text { nova y Robles Bayón, 2002; Roos, 2010). }\end{array}$ \\
& \\
\hline
\end{tabular}

${ }^{2}$ La razón por la cual podemos encontrar en la descripción de la enfermedad síntomas opuestos, como son la hipercinesia, hipocinesia, discinesia, acinesia y bradicinesia, es que el paciente experimenta sintomas motores hipercinéticos en el primer estadio y tiende a presentar una evolución hipocinética de manera progresiva hasta los estadios terminales. 
Sintomatologia comportamental

Irritabilidad, hipersexualidad, hipo-sexualidad, agresividad, desinhibición, psicosis, obsesiones, depresión, apatía, bipolaridad, alucinaciones y delirio (García de Yébenes, 2006; Peña-Casanova y Robles Bayón, 2002; Roos, 2010).

\section{Sintomatología lingüística}

Anomia, problemas en la interpretación de significados ambiguos, figurados o inferenciales y definición de conceptos, disgrafia, disartria, mutismo, disprosodia, perseveraciones, parafasias, escritura lenta, macrografia, problemas en la activación de conceptos y sus relaciones, y pérdida del lenguaje espontáneo (Arango-Lasprilla, Iglesias-Dorado y Lopera, 2003; Benitez Burraco, 2009; Diéguez-Vide y Peña-Casanova, 2011).

Tabla 1: Cuadro sintomatológico del huntington

\section{Metodología}

La consecución de este proyecto requiere el seguimiento de una serie de pasos. Concretamente, vamos a dividirlos en dos grandes bloques procedimentales: la recopilación de datos y el análisis de los mismos.

Comenzando por el primero de los bloques mencionados, el de la recopilación de datos, vemos necesaria una subdivisión en dos partes: la conversación espontánea y la conversación dirigida. Es cierto que hay autores que hacen uso tan solo de una de las dos; sin embargo, nos parece que el trabajo estaría incompleto. El motivo es la dificultad de registrar el déficit de un trastorno concreto. Como ya sabemos, el déficit es variable, por lo que el paciente no presenta exactamente los mismos sintomas todos los dias ni a todas horas, y sobre todo tampoco los presenta en todos los contextos. Pues bien, consideramos que al tener en cuenta ambos tipos de conversación, podremos recopilar una mayor cantidad de información del estado comunicativo del paciente.

Por un lado, siguiendo la metodología llevada a cabo por Gallardo Paúls y San Martín Sáez (2005), hemos tratado de obtener datos provenientes de una conversación espontánea con los dos pacientes seleccionados. El objetivo aquí es recopilar los datos deficitarios más habituales de la enfermedad, puesto que en los momentos más distendidos y en los que el paciente se siente más tranquilo, podemos localizar las afectaciones más cotidianas y llamativas. Muchos de estos síntomas no aparecen en las conversaciones guiadas.

En este caso, debemos destacar una serie de directrices fundamentales para lograr nuestro objetivo. Ante todo, la grabación debe realizarse en un lugar conocido y cómodo para el paciente, como puede ser su casa, la casa de un familiar o la consulta del neurólogo. Además, esta se realiza con su interlocutor clave, es decir, con aquella persona que más trato tenga con el paciente en su vida diaria. Algunas veces se trata de algún familiar, como el cónyuge, un hermano o hijo, y en otros casos son los cuidadores. La duración de la grabación consta aproximadamente de unos 15 minutos y los temas a tratar deben ser habituales en sus interacciones en la vida diaria. 
Como ejemplo, encontramos temas sobre lo que hacen en su tiempo libre, libros que hayan leído o películas y programas de televisión que hayan visto. Para esto, el interlocutor clave juega un papel que goza de ser esencial, ya que conoce al paciente, sus rutinas y gustos y es capaz de provocar una comunicación más espontánea, fluida y menos incómoda.

Por otro lado, para completar nuestro estudio de manera más eficaz hemos recopilado datos procedentes de una conversación guiada. Con este tipo de recursos es posible lograr un mayor control de la sintomatología de la enfermedad, debido a que hacemos uso de actividades programadas específicamente para conocer las destrezas lingüísticas del paciente en cada nivel de análisis. Pues bien, el instrumento que usamos en esta fase es uno de los más famosos test de evaluación del lenguaje: el Test Barcelona (1990). Con él procedemos a la evaluación de la producción y comprensión oral y escrita, a través de diferentes preguntas, órdenes y repeticiones de sonidos, sílabas, palabras y oraciones.

Finalmente, una vez obtenidos los datos descritos anteriormente, comenzaremos contrastándolos con aquellos aportados por la literatura científica para llegar a la descripción de la conducta lingüistica de cada paciente. Para ello, se tendrán en cuenta variables sociolingüísticas y médicas recogidas en la anamnesis y proporcionados por un neurólogo. Seguidamente, procederemos a la comparación de los resultados para cumplir con los objetivos propuestos en el apartado introductorio de este trabajo.

\section{ANÁlisis de los CASOS}

En este apartado, vamos a proceder con la exposición de los datos obtenidos empíricamente y con su análisis. En primer lugar, ofrecemos una tabla (Tabla 2), donde encontramos los datos más relevantes desde el punto de vista médico, y que también nos aportan información esencial desde la lingüística. Estos son: la fecha de nacimiento, que nos da información sobre qué variante de la enfermedad es manifestada; si la herencia es por vía materna o paterna; el año de diagnóstico, con el que podremos saber si la evolución se ha producido en un periodo de tiempo mayor o menor a lo esperado; las repeticiones de $\mathrm{CAG}$, que nos informan de si la progresión de la enfermedad será más agresiva, y el estadio de evolución en el que se encuentran los pacientes, a través del cual deberiamos saber la sintomatología esperada.

\begin{tabular}{|c|c|c|c|c|c|}
\hline Paciente & $\begin{array}{c}\text { Fecha de na- } \\
\text { cimiento }\end{array}$ & $\begin{array}{c}\text { Vía pa- } \\
\text { terna }\end{array}$ & $\begin{array}{c}\text { Año de diag- } \\
\text { nóstico }\end{array}$ & $\begin{array}{c}\text { Repeticiones } \\
\text { CAG }\end{array}$ & Estadio \\
\hline Paciente 1 & $10 / 06 / 1968$ & Sí & 2011 & 41 & II \\
\hline Paciente 2 & $16 / 08 / 1965$ & Sí & 2012 & 44 & II \\
\hline
\end{tabular}

Tabla 2: Variables médicas

En segundo lugar, podemos observar en la Tabla 3 las variables sociolingüísticas, que son fundamentales para el análisis lingüístico clínico. En ella 
vemos: la variedad dialectal tanto del paciente como de los padres, para poder distinguir entre rasgos que formen parte del idiolecto del individuo y rasgos deficitarios de la enfermedad; el nivel cultural y la ocupación previa del paciente, que resulta relevante para el control del vocabulario y estructuras aprendidas previamente al diagnóstico, y los idiomas, cuyo conocimiento es influyente en el desarrollo cognitivo y en el uso de la lengua materna.

\begin{tabular}{|c|c|c|c|c|c|}
\hline Paciente & $\begin{array}{l}\text { Variedad } \\
\text { dialectal }\end{array}$ & $\begin{array}{l}\text { Variedad de } \\
\text { los padres }\end{array}$ & $\begin{array}{l}\text { Nivel cultu- } \\
\text { ral o de es- } \\
\text { tudios }\end{array}$ & $\begin{array}{c}\text { Ocupación } \\
\text { previa }\end{array}$ & Idiomas \\
\hline Paciente 1 & $\begin{array}{l}\text { Andaluz oc- } \\
\text { cidental }\end{array}$ & $\begin{array}{c}\text { Andaluz oc- } \\
\text { cidental } \\
\text { (San Fer- } \\
\text { nando, Cá- } \\
\text { diz) + Cana- } \\
\text { rio }\end{array}$ & $8^{\circ}$ de EGB & $\begin{array}{l}\text { Conductor } \\
\text { de autobús }\end{array}$ & Español \\
\hline Paciente 2 & $\begin{array}{l}\text { Andaluz oc- } \\
\text { cidental }\end{array}$ & $\begin{array}{c}\text { Andaluz oc- } \\
\text { cidental } \\
\text { (San Fer- } \\
\text { nando, Cá- } \\
\text { diz) + Cana- } \\
\text { rio }\end{array}$ & $6^{\circ}$ de EGB & Ama de casa & Español \\
\hline
\end{tabular}

Tabla 3: Variables sociolingüísticas.

A continuación, procedemos a ofrecer los datos lingüísticos extraídos de la conversación espontánea y guiada, para poder establecer una comparativa junto con los resultados de la investigación de cada paciente.

\begin{tabular}{|c|c|}
\hline Producción oral & $\begin{array}{l}\text { Fonética: } \\
\text { - } \quad \text { Hradilalia } \\
\text { - Alargamientos vocálicos } \\
\text { - } \text { Adición de sonidos } \\
\text { - Disartria de sonidos } \\
\text { - } \quad \text { Fricatización de consonantes } \\
\text { - Parafasia fonémica } \\
\text { Gramática: } \\
\text { - Disgramatismo oracional } \\
\text { - Problemas de repetición en oraciones de más de } 8 \text { pala- } \\
\text { Semántica: } \\
\text { - Parafasia nominal } \\
\text { - Anomia }\end{array}$ \\
\hline
\end{tabular}




\begin{tabular}{|c|c|}
\hline & $\begin{array}{l}\text { - Imposibilidad de evocación categorial de más de } 3 \text { ele- } \\
\text { mentos } \\
\text { - Incapacidad de secuenciación inversa de meses del año } \\
\text { Pragmática y discurso: } \\
\text { - Inconclusión de frase }\end{array}$ \\
\hline Comprensión oral & $\begin{array}{l}\text { - Buena comprensión en conversación cotidiana. } \\
\text { - } \quad \text { Dificultad en responder a preguntas complejas. }\end{array}$ \\
\hline Producción escrita & $\begin{array}{ll}\text { - } & \text { Disortografia } \\
\text { - } & \text { Disgrafia } \\
\text { - } & \text { Omisiones de letras y artículos }\end{array}$ \\
\hline Comprensión escrita & $\begin{array}{ll}\text { - } & \text { Legastenia } \\
\text { - } & \text { Múltiples errores en preguntas escritas }\end{array}$ \\
\hline
\end{tabular}

Tabla 4: Sintomatología lingüística de Paciente 1.

\begin{tabular}{|c|c|}
\hline & Paciente 2 \\
\hline Producción oral & 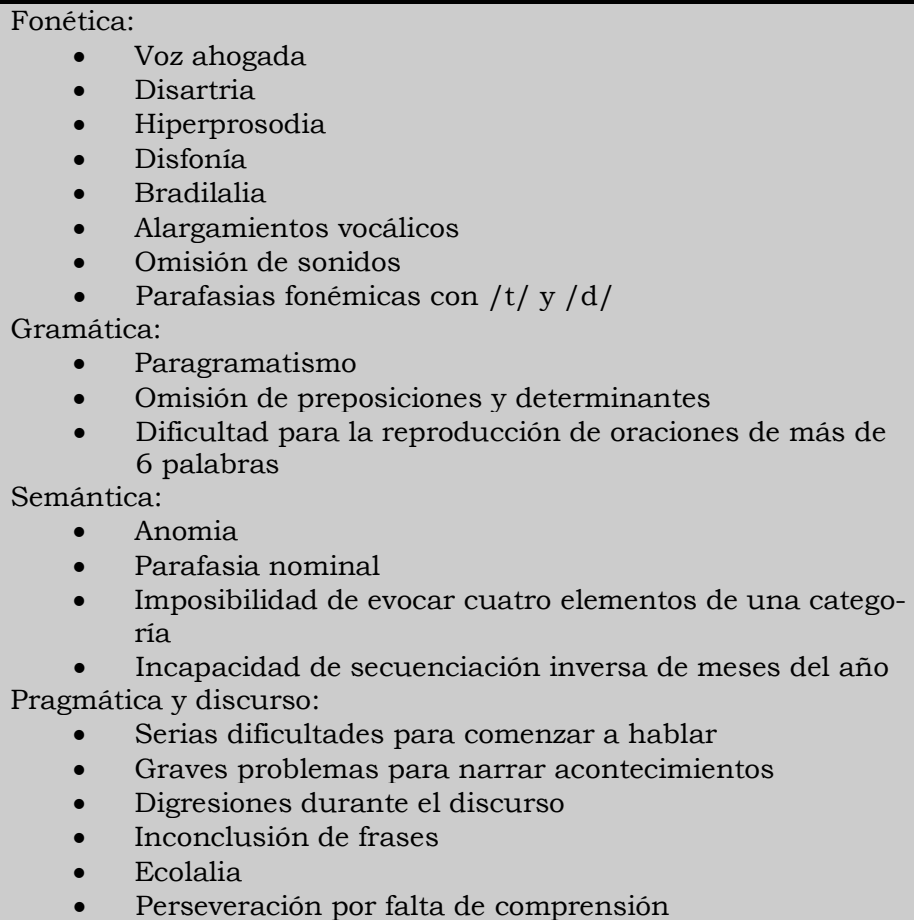 \\
\hline Comprensión oral & $\begin{array}{l}\text { - Dificultades en la comprensión de preguntas básicas } \\
\text { - Dificultad en la comprensión de órdenes }\end{array}$ \\
\hline Producción escrita & $\begin{array}{ll} & \text { Macrografia } \\
\text { - } & \text { Disortografia } \\
\text { - } & \text { Pisgrafia } \\
\text { - } & \text { Adición de letras } \\
\end{array}$ \\
\hline
\end{tabular}




\begin{tabular}{|l|ll|}
\hline & - Ausencia de verbos \\
\hline \multirow{3}{*}{ Comprensión escrita } & - Falta de concordancia gramatical \\
\hline & - Legastenia \\
& - Interrupciones en la lectura por pérdida \\
& - Múltiples errores en lectura de textos \\
\hline
\end{tabular}

Tabla 5: Sintomatología lingüística de paciente 2.

Como podemos observar, la selección de los dos pacientes presentados ha sido estratégica, debido a que son hermanos y, por ello, presentan unas variables médicas y sociolingüísticas muy parecidas. Sin embargo, en la sintomatología lingüística podemos ver claras diferencias.

En primer lugar, dentro de la producción oral, vemos claras semejanzas en las afectaciones fonéticas. Esto es debido a la gran afectación motora que es usual en el huntington. Como correlato a ello, cabía esperar que la articulación de sonidos y los rasgos prosódicos del discurso fuesen las afectaciones más notorias y comunes. No obstante, debemos destacar que, pese a que el paciente 1 cometa una mayor cantidad de errores diferentes, la diferencia es escasa y la capacidad de producción del paciente 2 es notablemente menor que la del paciente 1 .

En cuanto al componente gramatical, podemos ver diferentes afectaciones, como el disgramatismo en el paciente 1 y el paragramatismo en el paciente 2. Sin embargo, presentan un síntoma semejante, como es la dificultad de repetir oraciones largas. Dentro de ello, podemos apreciar que el paciente 2 se limita a oraciones con un máximo de 6 palabras, mientras que el paciente 1 logra producir hasta 8 palabras.

Siguiendo con los errores en la producción, hemos observado que semánticamente cometen los mismos tipos de fallos. Esta vez quien presenta una leve, aunque mayor afectación es el paciente 1, puesto que en las actividades de evocación de palabras por categorías no logra más de tres elementos, mientras que el paciente 2 se estanca en el cuarto. Sin embargo, el margen de error es poco significativo en este caso.

Para terminar con la producción oral, terminamos con el nivel pragmático y discursivo. En este parámetro es apreciable una gran diferencia entre ambos casos. Tanto el paciente 1 como el paciente 2 presentan frases inconclusas; sin embargo, el paciente 2 muestra muchas más dificultades para comenzar a hablar, narrar acontecimientos que conoce e incluso algo de ecolalia y perseveración de algún tema tratado en el vídeo. Estas afectaciones del paciente 2 limitan en consideración su capacidad comunicativa con su entorno.

En segundo lugar, hablamos de la comprensión oral. En este caso, aunque ambos pacientes tienen dificultades, la afectación del paciente 2 vuelve a ser notoriamente mayor. Concretamente, el paciente 1 se desenvuelve correctamente con preguntas y órdenes básicas, pero yerra en las de índole 
compleja. Frente a esto, el paciente 2 presenta grandes dificultades de comprensión durante la conversación cotidiana, además de problemas para entender preguntas básicas u órdenes simples.

En tercer lugar, aportamos tablas la producción escrita. Como vemos en la lista, volvemos a encontrar algunos sintomas comunes, como son la disgrafia y la disortografia o la paragrafia léxica. Sin embargo, además de las nombradas, el paciente 2 manifiesta una macrografia muy notoria, omite el verbo en las oraciones y tiene problemas de concordancia de género en palabras comunes.

Por último, también es posible apreciar la diferencia en la afectación de la comprensión escrita entre ambos pacientes, aunque no son idénticos. Por ejemplo, ambos realizan la lectura de forma muy lenta; sin embargo, el paciente 1 comete más errores en la respuesta a preguntas escritas. Por otro lado, el paciente 2 interrumpe más veces la lectura, confunde palabras con otras que tienen cierto parecido sonoro y solo yerra en la respuesta a preguntas escritas complejas.

En resumen, podemos afirmar que encontramos semejanzas en la sintomatología más básica del huntington; sin embargo, pese a que los dos pacientes presenten variables médicas y sociolingüísticas similares, la sintomatología es dispar. En la mayoría de los casos, el paciente 2 presenta una mayor afectación que el paciente 1 , de manera que la capacidad comunicativa del paciente 2 se ve notablemente más limitada que la del otro. Por ello, pese a que el diagnóstico sea idéntico, estamos ante dos casos que requerirían un programa de rehabilitación logopédica diferente.

\section{Conclusiones}

El presente trabajo se ha centrado en ofrecer un estudio de la sintomatologia lingüistica de dos pacientes que padecen la enfermedad de Huntington junto con una comparación entre ambos. Asimismo, los objetivos generales del trabajo han sido proporcionar datos lingüísticos de cada paciente que no aparecen en el diagnóstico neurológico, para que, de esta manera, sea posible conocer el comportamiento comunicativo de estos pacientes y establecer una comparativa entre los mismos con la intención de demostrar que la labor del lingüista es necesaria en los contextos clínicos.

Para tal finalidad, hemos presentado unos apartados que componen el marco teórico del trabajo. En el primero, hemos detallado brevemente en qué consiste la enfermedad y los síntomas más comunes desde las perspectivas médica y psicológica. También nos ha parecido especialmente relevante este apartado, puesto que se trata de una enfermedad rara y proporcionamos conocimiento útil para contextualizar el estudio.

Seguidamente, hemos redactado un apartado donde hemos expuesto la metodología que hemos seguido a lo largo de todo el proceso, tanto para la obtención del corpus de datos como para su análisis. Esto se ha realizado 
puesto que no concebimos un trabajo experimental sin la explicación de la metodología para su consecución por otros profesionales interesados.

Finalmente, hemos procedido a la presentación de los datos de cada paciente. Concretamente, hablamos de las variables médicas, sociolingüísticas y la sintomatología lingüística extraída del análisis de la grabación y el Test Barcelona. Tras esto, hemos elaborado una reflexión en la que se comparan ambos casos y se puede ver claramente que, pese a pertenecer al mismo estadio de evolución y, por lo tanto, compartir el mismo diagnóstico neurológico, las alteraciones lingüísticas de cada paciente son muy diferentes y, por tanto, su capacidad comunicativa. De este modo, podemos corroborar nuestra hipótesis sobre la necesidad de la labor de un lingüista en los contextos clinicos, ya que agilizaría y mejoraria la labor rehabilitadora del logopeda.

\section{REFERENCIAS}

ARANGO-LASPRILLA, J. C. et al. (2003): "Estudio neuropsicológico de la enfermedad de Huntington en familias de Antioquia, Colombia", Revista de neurología, 37, pp. 7-13.

ARANGO-LASPRILLA, J. C.; IGLESIAS-DORADO, J. y LOPERA, F. (2003): "Características clinicas y neuropsicológicas de la enfermedad de Huntington una revisión", Revista de Neurologia, 37 (8), pp. 758-765.

BENÍTEZ BURRACO, A. (2008): Genes y lenguaje. Aspectos ontogenéticos, filogenéticos y cognitivos, Barcelona: Editorial Reverté.

BENÍTEZ BURRACO, A. (2009): "Enfermedad de Huntington: fundamentos moleculares e implicaciones para una caracterización de los mecanismos neuronales responsables del procesamiento lingüístico", Revista de neurologia, 48 (2), pp. 75-84.

CUBA, J. M. y TORRES, L. (1990): "Estudio de una familia con corea de Huntington en Cañete", Revista de Neuro-Psiquiatría, 53, pp. 94-102.

FRANCH VALVERDE, J. I. (1993): La enfermedad de Huntington, Va- lladolid: Servicio de Publicaciones de la Universidad de Valladolid.

GALLARDO PAÚLS, B. y SAN MARTÍN SÁEZ, J. (2005): Afasia fluente. Materiales para su estudio, (Volumen 1 del corpus PerLA), Valencia: Universitat de València.

GARCÍA DE YÉBENES, J. (2006): "La demencia de la enfermedad de Huntington", Alberca, S. y López-Pousa, S. (dir.): Enfermedad de Alzheimer $y$ otras demencias, Madrid: Editorial Médica Panamericana, pp. 381394.

HESSE, H. et al. (2006): "Enfermedad de Huntington. Reporte de dos casos en una familia hondureña y revisión de la literatura", Revista Médica Hondureña, 74, pp. 195-200.

HINZEN, W. et al. (2018): “A systematic linguistic profile of spontaneous narrative speech in presymptomatic and early stage Huntington's disease", Cortex, 100, pp. 71-83.

KASPER, D. L. et al. (2017, 19 edic.): Harrison. Manual de medicina, México D. F: McGraw-Hill Interamericana Editores. 
PEÑA-CASANOVA, J. (1990): Programa integrado de exploración neuropsicológica "Test Barcelona", Barcelona: Masson.

PEÑA-CASANOVA, J. y ROBLES BAYÓN, A. (coord.) (2002): Guías en demencias. Conceptos, criterios y recomendaciones para el estudio del paciente con demencia, Barcelona: Masson.

ROOS, R. (2010): "Huntington's disease: a clinical review", Orphanet Journal of Rare Diseases, 5 (40). Disponible en: http:/ /www.biomedcentral.com/content/pdf/17501172-5-40.pdf (Fecha de consulta: $12 / 12 / 2018$ ).

SARASON, I. G. y SARASON, B. G. (2006): Psicopatología. Psicología anormal: el problema de la conducta inadaptada, México: Pearson Educación.

SCRIMGEOUR, E. M. (1980): "Huntington's disease in two New Britain families", Journal of Medical Genetics, 17, pp. 197202. 